07,04

\title{
Исследование тонкой структуры канала наносекундного пробоя в хлориде калия
}

\author{
(C) Р.В. Емлин, И.Ф. Пунанов \\ Институт электрофизики УрО РАН, \\ Екатеринбург, Россия \\ ฯ E-mail: ivan.punanov@gmail.com
}

(Поступила в Редакцию 31 августа 2016 г.)

Получена картина тонкой структуры канала пробоя с положительного электрода в монокристалле $\mathrm{KCl}$ в режиме многоимпульсного воздействия при напряжении до $140 \mathrm{kV}$. Определены размеры и форма пробойных структур в зависимости от действующего напряжения. Рассчитаны скорости распространения вершины трещины, формирующей пробойную структуру, а также давление в канале пробоя. Показано, что в определенных условиях структура механических разрушений вблизи канала пробоя сохраняется даже после воздействия нескольких десятков импульсов.

Работа поддержана Российским фондом фундаментальных исследований (грант № 11-08-01003).

DOI: 10.21883/FTT.2017.08.44755.336

\section{1. Введение}

Формирование канала пробоя является наименее изученным этапом процесса электрического пробоя твердых диэлектриков, однако именно этот этап является наиболее интересным с фундаментальной точки зрения. Изучение возникшей в результате пробоя структуры электрического и механического разрушения может дать информацию об элементарных процессах, идущих в канале пробоя. В работах, в которых есть указания на существование внутренней структуры канала [1-4], речь идет, как правило, о каналах, возникших в результате действия микросекундных импульсов. В диапазоне напряжений $100-200 \mathrm{kV}$ скорости развития канала в кристаллах составляют $\sim 10^{6}-10^{7} \mathrm{~cm} / \mathrm{s}$ [5], так что за время действия импульса длительностью $\sim 10^{-7}-10^{-6} \mathrm{~s}$ пробивается образец длиной порядка нескольких сантиметров, а процессы механических разрушений маскируют тонкую структуру канала. Поскольку длительность импульсов напряжения в этих работах была гораздо дольше самого процесса формирования канала, либо соизмерима с ней, в таком режиме трудно было пронаблюдать изменения в канале, которые вызваны подъемами и спадами напряжения на фронтах импульса.

В режиме наносекундных импульсов $\left(\sim 10^{-9}-10^{-8} \mathrm{~s}\right)$, напротив, за время действия отдельного импульса канал не успевает прорасти на всю длину образца с размерами порядка сантиметра. Для завершения процесса пробоя требуется время, равное времени действия нескольких импульсов. Можно предположить, что в относительно толстых образцах кристаллов (более $10 \mathrm{~mm}$ ) при воздействии ультракоротких импульсов будут возникать пробойные структуры, сформированные несколькими отдельными импульсами из последовательности. Результатов по исследованию тонкой структуры каналов наносекундного пробоя в литературе практически нет, за исключением нескольких работ [6-8].

Для наблюдения тонкой структуры канала пробоя хорошим модельным объектом является кристалл $\mathrm{KCl}$, поскольку он всесторонне изучен, имеет простую кубическую структуру, легко раскалывается для изготовления образцов требуемых размеров. Ранее получено [9], что при напряжении $140 \mathrm{kV}$ и длительности импульса 8 ns скорость развития канала в $\mathrm{KCl}$ для относительно тонких $(1-3 \mathrm{~mm})$ образцов составляет $(3-5) \cdot 10^{7} \mathrm{~cm} / \mathrm{s}[9]$. Однако, учитывая, что время развития канала растет с увеличением толщины кристалла нелинейно [10], в относительно толстом образце при тех же параметрах начального импульса можно ожидать среднюю скорость развития канала в несколько раз меньше, то есть такую, при которой за время действия одного из последовательности отраженных импульсов канал продвинется на расстояние порядка сотен микрометров. Такой режим дает возможность получить микроструктуру, отражающую динамику развития канала с наносекундным разрешением.

\section{2. Эксперимент}

Для экспериментов использовался генератор наносекундных импульсов, представляющий собой коаксиальную формирующую линию с импедансом $Z=50 \Omega$, заряжаемую от трансформатора Тесла. Электрическая емкость линии равнялась $80 \mathrm{pF}$, а запасаемая энергия $0.8 \mathrm{~J}$. Формирующая линия замыкается на передающую линию неуправляемым газовым разрядником-обострителем. Напряжение срабатывания разрядника равно $140 \mathrm{kV}$. Длительность импульса на согласованной нагрузке составляет $8 \mathrm{~ns}$, длительность переднего фронта импульса менее $0.5 \mathrm{~ns}$. Сходные по конструкции высоковольтные наносекундные генераторы описаны в [11]. 


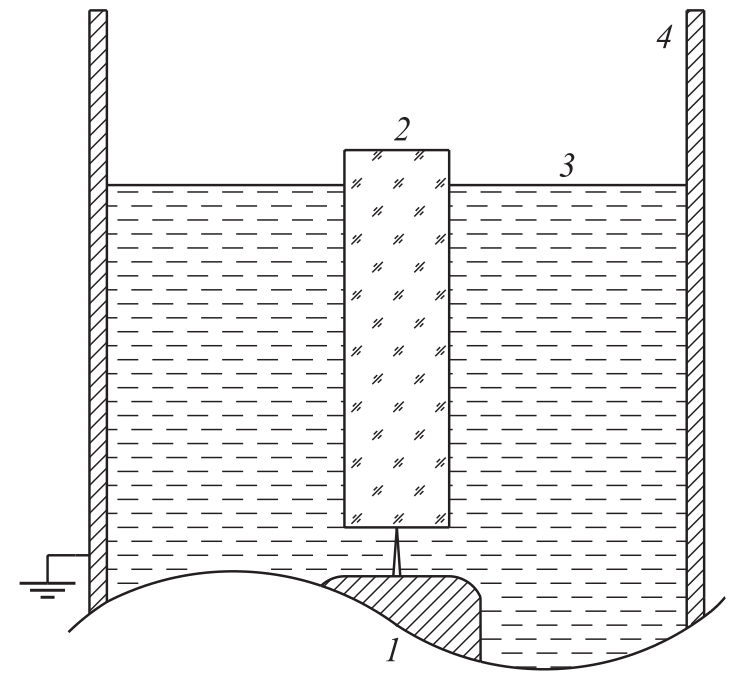

Рис. 1. Эскиз пробойной ячейки: 1 - высоковольтный электрод (анод); 2 - образец; 3 - трансформаторное масло; 4 - заземленный корпус (катод).

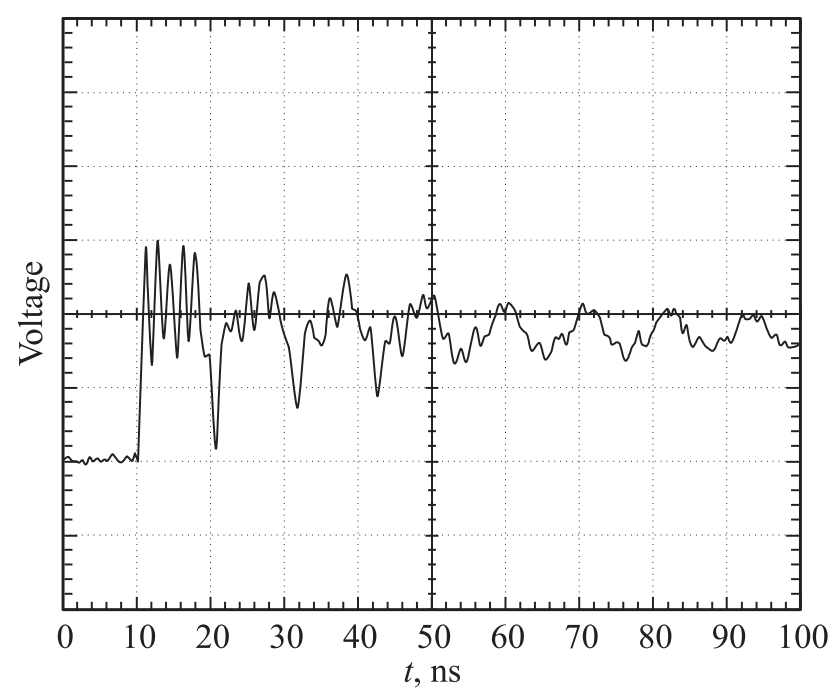

Рис. 2. Осциллограмма последовательности импульсов на игловом электроде пробойной ячейки.

Образец монокристаллического $\mathrm{KCl}$ размерами $10 \times 10 \times 30 \mathrm{~mm}$ помещался в ячейку, заполненную трансформаторным маслом (рис. 1). Нижняя грань образца контактировала с игловым анодом в масле, в то время как верхняя грань контактировала с воздухом [12].

Пробойная ячейка соединена с разрядником через передающую линию длиной $30 \mathrm{~cm}$, заполненную трансформаторным маслом. Введение такой относительно длинной передающей линии позволило сформировать цуг хорошо разделенных импульсов с постепенно уменьшающейся амплитудой (см. рис. 2), регистрируемый осциллографом Tektronix TDS 644В. Этот цуг возникает при отражении импульса от границы разомкнутой линии передачи, которую образует часть тракта генератора от разрядника до вершины развивающегося канала пробоя. Амплитуда импульсов от $140 \mathrm{kV}$ последовательно уменьшается из-за потерь энергии при отражениях в формирующей линии генератора и из-за расхода энергии на образование канала пробоя. Число отражений может достигать нескольких десятков в зависимости от длины образца.

Изображенная на рис. 2 последовательность высоковольтных импульсов эквивалентна электрическим колебаниям с частотой порядка $50 \mathrm{MHz}$. При такой частоте слой воздуха между кристаллом и заземленным металлическим корпусом генератора играет роль высокоомного балластного резистора с широкой полосой пропускания, ограничивающего ток в заключительной стадии пробоя, когда генератор разряжается через малое сопротивление плазменного канала и сопротивление этого слоя при выходе канала на верхнюю грань образца. Благодаря этому ограничению тока в кристалле сохраняется картина разрушений, возникших в режиме многоимпульсного воздействия. На рис. 3, $a$ изображены участки полученного в эксперименте канала пробоя в образце KCl. Типичный элемент картины пробоя при большом увеличении показан на рис. $3, c$.

\section{3. Обсуждение результатов}

Из рис. 3, $a$ видно, что область пробоя представляет собой не просто цилиндрический канал постоянного сечения, а последовательность элементов со сложной геометрией. Можно выделить область „тонкой“ части канала диаметром около $10 \mu \mathrm{m}$ и „толстой“ части диаметром $50 \mu \mathrm{m}$. По обе стороны от „тонкой“ части канала в плоскости раскола видна относительно гладкая область, близкая по форме к окружности диаметром $500 \mu \mathrm{m}$. На внешних границах этих областей наблюдаются микрокапли $(3-5 \mu \mathrm{m})$ сконденсировавшегося из плазменного состояния вещества канала.

На основании результатов измерения скорости развития канала пробоя в щелочно-галоидных кристаллах, полученных в сходных условиях [5,8-10], предполагается, что каждый такой элемент пробойной структуры образовался за время действия одного импульса длительностью $8 \mathrm{~ns}$. Это позволяет использовать характерный размер и форму элемента пробойной структуры для временной привязки при рассмотрении сопутствующих процессов.

Экспериментальные данные, полученные в $[6,7,13]$, дают еще больше оснований для такой интерпретации вида наблюдаемой пробойной структуры. В работе [13] введено понятие критической радиальной напряженности поля $E_{\text {cr }}$, обеспечивающей существование канала анодного пробоя. В соответствии с этой идеей, постоянство скорости распространения канала пробоя с анода и переносимого заряда при изменении мгновенных значений параметров импульса $d U / d t$ обеспечивается за счет изменения площади сечения канала пробоя, причем 

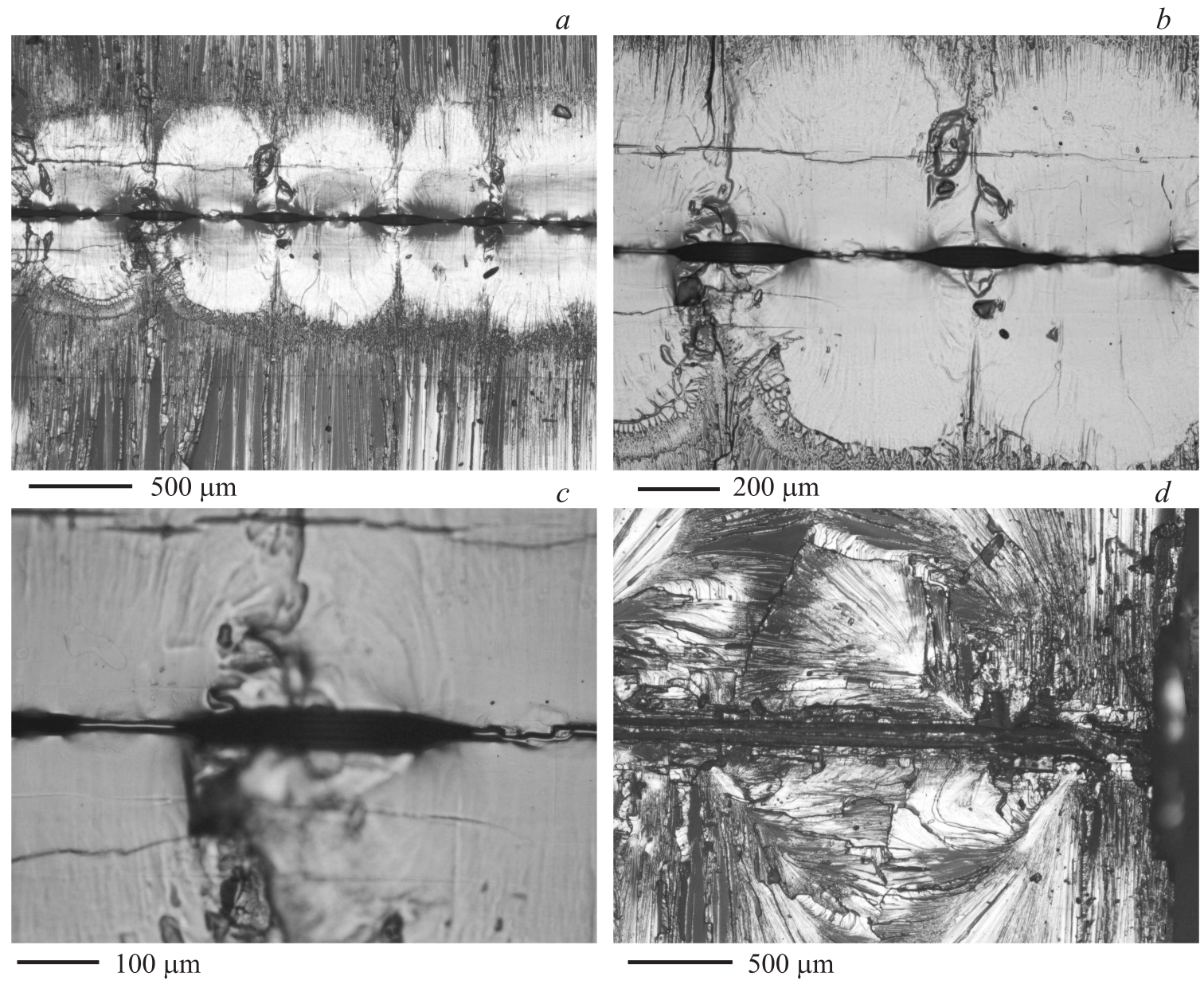

Рис. 3. Участок канала пробоя в $\mathrm{KCl}(a, b, c)$; канал пробоя вблизи выхода на поверхность кристалла $(d)$.

радиус канала изменяется пропорционально мгновенным значениям напряжения [6].

Очевидно, что не приходится говорить о постоянстве скорости развития канала при воздействии длинной последовательности импульсов, но в пределах действия нескольких импульсов из последовательности это условие может выполняться, что дает основание считать, что периодические сужения и расширения канала представляют собой визуализацию изменения напряжения на пробойном промежутке, то есть их диаметр коррелирует с мгновенными значениями действующего напряжения.

Последовательность процессов в разрядном промежутке, приводящая к возникновению картины пробоя, изображенной на рис. 3, $a$, представляется следующей. Приходящий фронт импульса напряжения (0.5 ns) формирует „тонкую“ часть канала диаметром около $10 \mu \mathrm{m}$, которая постепенно расширяется до стационарного диаметра около $50 \mu \mathrm{m}$ при выходе напряжения на максимальное значение (плоская часть импульса). При спаде напряжения на заднем фронте импульса, отраженного от проводящей вершины канала, диаметр снова уменьшается до значения около $10 \mu \mathrm{m}$. С приходом последующего импульса этот процесс происходит вновь, формируя следующий элемент картины пробоя. То, что области с круговыми локальными трещинами, находящимися по обе стороны от „тонких“ участков канала в плоскости раскола, возникли в результате действия фронтов импульса, подтверждается фотографиями области канала со стороны катода (рис. 3,d), где канал имеет практически цилиндрическую форму, а область ближнего разрушения вокруг канала, подобная той, что изображена на рис. $3, b$, отсутствует. В той части канала, находившейся ближе к катоду, действовавшее напряжение уже не имело больших значений $d U / d t$, поэтому канал формировался практически равномерно (см. рис. 3,d). Следует отметить, что четкая граница и сохранение формы каждой из локальных трещин свидетельствуют о том, что последующие импульсы напряжения не изменяют их форму и размер существенным образом.

Сохранение картины локального разрушения происходит, вероятно, по следующим причинам. После расширения плазмы канала и конденсации ее в вершине трещины устье трещины смыкается за время, равное отношению радиуса канала к скорости звука в веществе: $t=5 \cdot 10^{-6} \mathrm{~m} / 4.5 \cdot 10^{3} \mathrm{~m} / \mathrm{s} \approx 10^{-9} \mathrm{~s}$. Поскольку плазменные электроны „вытягиваются“ электрическим 
полем в канал и уходят на анод, в стенках трещины происходит „замораживание“ положительного заряда и соответствующего ему достаточно высокого положительного потенциала. Давление остаточной высокопроводящей плазмы в канале невелико, поскольку большая часть испарившегося вещества ушла в вершину трещины и сконденсировалась в ней (рис. $3, b$ ). Прохождение тока формирования следующего за рассматриваемым участка канала (величина его порядка 10 А) $[14,15]$ не вызывает заметного нагрева и появления более высоких давлений, что способствует сохранению детальной картины механических дефектов.

Вид полученной пробойной структуры, результаты измерений и расчеты указывают, что в условиях проведенного эксперимента процесс формирования канала пробоя под действием каждого приходящего импульса из последовательности происходит непосредственно в момент прохождения фронта пробоя, а наблюдаемая на фотографиях картина разрушения представляет собой визуализацию действия напряжения на пробойном промежутке. Особенно подчеркивает эти выводы наличие участков „сужения“ канала, соответствующих моментам времени, когда из-за отражения пришедшего наносекундного импульса напряженность поля на растущей вершине канала понижается до минимально необходимой для электрического разрушения вещества образца [8]. Подтверждением того, что такой режим может реализоваться, служит наблюдение удлинения области свечения в канале пробоя при подаче на образец единичных импульсов амплитудой $70-80 \mathrm{kV}$ в режиме неполного пробоя. Приложение такого же по длительности единичного импульса к этому же образцу через некоторое время приводило к прорастанию канала вглубь примерно на такое же расстояние. Это говорит о том, что изменения в образце, созданные первым импульсом, таковы, что второй приходящий импульс не встречает существенного сопротивления на этом участке и, следовательно, энергия на нем не расходуется, а вся идет на образование следующего участка канала.

Характерное расстояние, на котором в плоскости раскола наблюдается разрушение кристалла, вызванное непосредственно пробоем, составляет около $500 \mu \mathrm{m}$ (см. рис. $3, a, b)$. В предположении, что фронт разрушения распространялся со скоростью продольного звука, равной в хлориде калия $4.5 \cdot 10^{5} \mathrm{~cm} / \mathrm{s}$ для направления $\langle 100\rangle[16]$, для преодоления этого расстояния потребовалось бы $1.1 \cdot 10^{-7} \mathrm{~s}$. За такое время следующие приходящие импульсы разрушили бы тонкую структуру канала. Из рис. 3, $a$ видно, что структура разрушения регулярная, а характерные размеры области разрушения вокруг канала $(500 \mu \mathrm{m})$ и области между „тонкими“ частями канала близки. Это позволяет предположить, что приканальная область (рис. $3, b)$ и элемент канала сформировались за одно и то же время, равное времени действия импульса высокого напряжения, т.е. $8 \mathrm{~ns}$. Для этого скорость формирования трещины должна достигать десятков километров в секунду.
Известно, что трещина в кристалле может распространяться со скоростью, превышающей скорость звука в рассматриваемом веществе, если энергия, введенная в кристалл, много больше энергии, затрачиваемой на раскалывание, и время воздействия мало [17]. Энергия образования свободной поверхности для $\mathrm{KCl}$ равна $1.1 \cdot 10^{-5} \mathrm{~J} / \mathrm{cm}^{2}[18]$. Оценивая сверху энергию, затраченную на раскалывание, примем, что общая площадь растрескивания составляет $6 \mathrm{~cm}^{2}$ (трещины образовались в двух перпендикулярных плоскостях, пересекающихся по каналу, ширина образца около $10 \mathrm{~mm}$, длина $30 \mathrm{~mm}$ ), получим величину энергии около $6.6 \cdot 10^{-5} \mathrm{~J}$.

Энергию, введенную в кристалл, можно снизу оценить по объему вещества, переработанного в плазму (в приближении однократно заряженных ионов):

$$
W_{\text {ch }}=N U_{i}=n V U_{i}=N_{A} \rho\left(\pi r^{2} l\right) U_{i} / M .
$$

Здесь $N$ - общее число молекул в рассматриваемом объеме, $U_{i}$ - потенциал ионизации, $n-$ концентрация, $V$ - объем элемента канала, $N_{A}$ - число Авогадро, $M$ - молекулярная масса, $r$ - радиус канала, $\rho-$ плотность вещества, $l$ - длина элемента канала.

Взяв наибольший потенциал ионизации (в данном случае для хлора), получаем $W_{\text {ch }}=1.34 \cdot 10^{-2} \mathrm{~J}$. Таким образом, энергия образования одного элемента пробойной структуры по крайней мере на два порядка превышает верхнюю оценку энергии, необходимую на создание трещины. Следовательно, для отдельно взятого элемента пробойной структуры энергетические условия из $[17,18]$ выполняются (а также условие малого времени воздействия), что подтверждает гипотезу о том, что трещина распространялась под воздействием удара фронта плазменного сгустка со скоростью порядка $50 \mathrm{~km} / \mathrm{s}$. Плазменный сгусток поставляет энергию на образование свежей поверхности в область вершины трещины. Это согласуется с результатом авторов $[17,18]$, исследовавших лазерный пробой в $\mathrm{KCl}$ при сравнимой мощности энерговвода. Также это согласуется с результатами нашей работы [19], где получены скорости порядка $20-100 \mathrm{~km} / \mathrm{s}$ у плазменных сгустков, истекающих из канала пробоя в $\mathrm{KCl}$ при выходе его на поверхность образца, а также генерируемых при поверхностном перекрытии монокристаллов $\mathrm{KCl}$ в вакууме [20].

Значения скорости распространения канала пробоя и геометрические параметры можно использовать для расчетов и оценок таких характеристик, как давление плазменного вещества в канале и радиальная напряженность электрического поля в кристалле на границе проводящего канала.

Давление в канале пробоя можно оценить, рассмотрев канал как кольцо, нагруженное по внутреннему контуру равномерно распределенными нормальными усилиями [21], как это сделано в работе [22]. Пренебрегая коэффициентами, связанными с анизотропией объекта, а также учитывая, что отношение радиуса канала к радиусу образца $(5 \mathrm{~mm})$ много меньше отношения радиуса 
канала к глубине трещины, получаем формулу:

$$
\begin{aligned}
P & =\sigma_{\max }\left(1-\left(r_{0} / r_{1}\right)^{2}\right) /\left(\left(r_{1} / r_{2}\right)^{2}\left(1+\left(r_{2} / r_{1}\right)^{2}\right)\right) \\
& \approx \sigma_{\max }\left(r_{1} / r_{0}\right)^{2} .
\end{aligned}
$$

Здесь $\sigma_{\max }-$ прочность на разрыв, $r_{0}-$ радиус канала, $r_{1}$ - глубина трещины, $r_{2}$ - радиус образца.

Сделаем оценку давления для канала, изображенного на рис. $3, b$. Из фотографии видно, что той части канала, радиус которой равен $5 \mu \mathrm{m}$, соответствует трещина (область ближнего разрушения) глубиной около $500 \mu \mathrm{m}$. Широкой части канала, радиус которой равен $25 \mu \mathrm{m}$, соответствует трещина глубиной около $250 \mu \mathrm{m}$. Статическая прочность на разрыв (предельное нормальное механическое напряжение) $\sigma_{\max }$ кристалла $\mathrm{KCl}$ для кристаллографической плоскости $\{100\}$ составляет $5 \cdot 10^{6} \mathrm{~Pa}$. Таким образом, рассчитанное давление равно $5 \cdot 10^{10} \mathrm{~Pa}$ и $5 \cdot 10^{8} \mathrm{~Pa}$ для участков канала радиусами соответственно 5 и $25 \mu \mathrm{m}$. По порядку величины это совпадает с результатами работы [21], где на основании экспериментальных данных о геометрических параметрах трещин при анодном пробое в $\mathrm{KCl}$ импульсами напряжения $220 \mathrm{kV}$ давление плазмы в канале пробоя в процессе его формирования оценено как $(6-24) \cdot 10^{10} \mathrm{~Pa}$.

Поскольку временные масштабы развития процесса наносекундного пробоя диэлектрика лежат в диапазоне $10^{-10}-10^{-9} \mathrm{~s}$, физический механизм пробоя должен обеспечивать генерацию плазмы свободных электронов, атомов и ионов за эти достаточно малые времена. Таким механизмом могут быть туннельные переходы электронов размытой вершины валентной зоны в зону проводимости [23]. В рассматриваемом случае распространение фронта пробоя представляет собой квазистационарный процесс образования плазменного канала, имеющий определенную скорость. Скорость этого процесса есть скорость перемещения фронта фазового перехода в области вершины канала. Эта скорость коррелирует со скоростью дрейфа электронов к аноду, определяемая как подвижность носителей, умноженная на напряженность поля в некоторой области перед вершиной канала. Оценка напряженности поля в системе „гиперболическое острие-плоскость“" дает $4 \cdot 10^{7} \mathrm{~V} / \mathrm{cm}$. При такой напряженности поля скорость порядка $5 \cdot 10^{6} \mathrm{~cm} / \mathrm{s}$ соответствует средней подвижности носителей около $0.1-1 \mathrm{~cm}^{2} / \mathrm{V} \cdot \mathrm{s}$, что является вполне разумной оценкой для подвижности свободных электронов в щелочногалоидных монокристаллах.

\section{Список литературы}

[1] Н.М. Торбин. В сб.: Физика диэлектриков. Тр. II Всесоюзн. конф. (1958). Изд-во АН СССР, М. (1960). С. 415.

[2] Дж.Б. Беркс, Дж.Г. Шулман. Прогресс в области диэлектриков. Госэнергоиздат, М., Л. (1962). Т. І. С. 80.

[3] А.А. Воробьев, Г.А. Воробьев. Электрический пробой и разрушение твердых диэлектриков. Высш. шк., М. (1966). C. 122.
[4] Ю.Н. Вершинин, В.Г. Герман. В сб.: Электрофизические проблемы применения твердых и комбинированных диэлектриков в технике высоких напряжений. Наука, Новосибирск (1974). С. 5.

[5] Ю.Н. Вершинин, Р.В. Емлин, С.О. Чолах, В.Г. Шпак, В.А. Белоглазов. ПЖТФ 16, 6, 49 (1990).

[6] Ю.Н. Вершинин, А.А. Подрезов, С.В. Барахвостов. В сб.: Тез. докл. VI науч. шк. „Физика импульсных воздействий на конденсированные среды“. ИМПиТ АН Украины, Николаев (1993). С. 24.

[7] Ю.Н. Вершинин. Электронно-тепловые и детонационные процессы в диэлектриках. УрО РАН, Екатеринбург (2000). C. 214.

[8] И.Ф. Пунанов, Р.В. Емлин, А.С. Гилёв, С.О. Чолах. ФТТ 53, 5, 875 (2011).

[9] И.Ф. Пунанов, Р.В. Емлин, В.Д. Куликов, С.О. Чолах. ЖТФ 84, 4, 35 (2014).

[10] И.Ф. Пунанов, Р.В. Емлин, П.А. Морозов, С.О. Чолах. Изв. вузов. Физика 55, 2, 57 (2012).

[11] Г.А. Месяц. Импульсная энергетика и электроника. Наука, M. (2004). 704 c.

[12] Yu.N. Vershinin, A.A. Podrezov, S.V. Barakhvostov, A.S. Pleshanov, I.B. Rogdestvensky. Proc. $3^{\text {rd }}$ Int. Conf. Properties and Applications of Dielectric Materials. Tokyo (1991). P. 1189.

[13] Ю.Н. Вершинин. В сб.: Материалы Междунар. научнотехн. конф. по физике твердых диэлектриков „Диэлектрики-97“, СПб. (1997). С. 147.

[14] Р.В. Емлин, В.А. Белоглазов. В сб.: Тез. докл. VI науч. шк. „Физика импульсных воздействий на конденсированные среды“. ИМПиТ АН Украины, Николаев (1993). С. 195.

[15] I.F. Punanov, R.V. Emlin, V.D. Kulikov, S.O. Cholakh. Изв. вузов. Физика 55, 12/3, 48 (2012).

[16] А.А. Блистанов, В.С. Бондаренко, Н.В. Переломова, Ф.Н. Стрижевская, В.В. Чкалова, М.П. Шаскольская. Акустические кристаллы. Наука, М. (1982). 632 с.

[17] S. Winkler, D.A. Shockey, D.R. Curran. Int. J. Fracture Mechanics 6, 2, 151 (1970).

[18] D.R. Curran, D.A. Shockey, S. Winkler. Int. J. Fracture Mechanics 6, 3, 271 (1970).

[19] I.F. Punanov, R.V. Emlin, P.A. Morozov, S.O. Cholakh. Proc. of the IEEE Int. Pulsed Power and Plasma Science Conference. San Francisco (2013). P. 790.

[20] I.F. Punanov, R.V. Emlin, P.A. Morozov, S.O. Cholakh. Abstracts of the IEEE Int. Conf. on Plasma Science and High Power Particle Beams. Washington (2014). P. 1.

[21] С.Г. Лехницкий. Анизотропные пластинки. ГИТТЛ, М., Л. (1947). С. 137.

[22] В.Д. Соловей, В.Л. Колмогоров, Ю.Н. Вершинин. ПМТФ 43, 3, 24 (2002).

[23] В.Д. Куликов. ЖТФ 82, 2, 35 (2012). 